Disponible on-line en:

http://revistalogopedia.uclm.es

UNIUERSIDADDE Revista de Investigación en Logopedia 1 (2015) 60-70.

\title{
Características formales y transparencia de los símbolos pictográficos de ARASAAC
}

\author{
Francisco Cabello y Elisabetta Bertola
}

Universidad de Murcia, España

\begin{abstract}
Resumen
Se examina la iconicidad y transparencia de los símbolos pictográficos de ARASAAC, y se compara con la de otros conjuntos de signos ya establecidos (SPC y Bliss). Participaron 34 estudiantes universitarios, expuestos a una tarea en la que se presentaban 114 símbolos correspondientes a cuatro categorías gramaticales (nombres, verbos, adjetivos y símbolos lingüísticos) y se valoraba su relación con el significado mediante una escala de 7 puntos. Los resultados muestran que los pictogramas ARASAAC tienen un adecuado grado de transparencia e iconicidad, incluso mayor que la de los pictogramas SPC, aunque hay variaciones significativas en función de la categoría gramatical.
\end{abstract}

Palabras clave: ARASAAC; Comunicación alternativa; Iconicidad; Pictogramas; Transparencia.

\section{Formal characteristics and transparency of the ARASAAC pictographic symbols}

\begin{abstract}
The iconicity and transparency properties of the ARASAAC pictographic symbol set is examined, and compared to that of other established symbol sets (SPC and Blissymbols). 34 undergraduate students participated in the study, and were exposed to a task in which 114 symbols were presented from four grammatical categories (nouns, verbs, adjectives and linguistic symbols) and their relation with their meaning was determined using a 7-point scale. Results indicate that pictograms from the ARASAAC set have an adequate degree of iconicity and transparency, even higher than symbols from the SPC set, although significative emerged related to the different grammatical categories.
\end{abstract}

Keywords: Alternative communication; ARASAAC; Iconicity; Pictograms; Transparency.

Correspondencia con los autores: fcabello@um.es

Recibido 9 Abril 2015. Primera revisión 4 de Mayo de 2015. Aceptado 18 Mayo de 2015. 


\section{Introducción}

Los símbolos pictográficos, en los que se representa gráficamente de manera sencilla el referente que quieren significar, son uno de los recursos más utilizados en la comunicación aumentativa y alternativa (Beukelman y Mirenda, 2013), por lo que no es de extrañar la existencia de numerosos conjuntos de pictogramas como los de SPC, Bliss, PicSyms, Rebus o Pics for PECS. Si bien los símbolos de estos conjuntos pueden describirse de acuerdo a distintas dimensiones como por ejemplo el realismo, la ambigüedad o la eficiencia (ver Fuller, Lloyd y Stratton, 1997; Schlosser y Sigafoos, 2002; Wilkinson y Jagaroo, 2004), la característica que ha recibido más atención es la iconicidad, entendida como "la asociación que el individuo hace entre un símbolo y su referente" y que, de acuerdo con Fuller y Lloyd (1997), puede conceptualizarse como un continuo. En un extremo del mismo estarían los símbolos transparentes, cuyo significado es sugerido fácilmente y por tanto puede ser determinado por cualquier persona, mientras que en el otro estarían los símbolos opacos, aquellos signos abstractos que tienen un significado difícil de entender; entre ambos extremos se encontrarían los símbolos translúcidos, en los que el significado no es fácilmente accesible pero en los que se puede percibir una cierta relación.

Existe un amplio consenso sobre esta "hipótesis de la iconicidad", y lo que sería más importante, hay múltiples evidencias que señalan que la iconicidad de un pictograma puede facilitar o dificultar el aprendizaje y retención de la relación con su referente, de manera que a mayor transparencia mejor aprendizaje (por ejemplo, Angermeier, Schlossoer, Luiselli, Harrington y Carter, 2008; Fuller, 1997; Hetzroni, Quist y Lloyd, 2002; Hulburt, Iwata y Green , 1982; Koul, Schlosser y Sancibrian, 2001; Luftig y Bersani, 1985; Schlosser et al., 2012).

Por este motivo, no es de extrañar que numerosos trabajos hayan explorado las características de iconicidad de los distintos conjuntos de pictogramas a los que se aludía más arriba, tanto en sujetos con desarrollo normal como con algún tipo de discapacidad (Bloomberg, Karlan y Lloyd, 1990; Mirenda y Locke, 1989; Mizuko, 1987; Mizuko y Reichle, 1988; Musselwhite y Ruscello, 1983). Por ejemplo, Bloomberg et al. (1990) investigaron la transparencia de cinco conjuntos de símbolos: Bliss, Picsyms, PIC, SPC y Rebus. Los participantes señalaron el nivel de iconicidad de los distintos símbolos empleando para ello una escala tipo Likert de 7 puntos, encontrándose que los sistemas SPC y Rebus obtuvieron las mayores puntuaciones de transparencia. 
A través de este tipo de trabajos se ha encontrado de manera consistente que los distintos conjuntos de pictogramas tienen características de iconicidad muy diferentes, de manera que por ejemplo el SPC se considera el sistema más transparente mientras que el Bliss es el más opaco (para una revisión, puede consultarse Schlosser y Sigafoos, 2002).

En esta investigación se analiza de manera preliminar la iconicidad y transparencia de los símbolos pictográficos de ARASAAC, consistentes en un conjunto de símbolos gráficos, tanto en blanco y negro como en color, que con un estilo sencillo y homogéneo cubren un amplísimo vocabulario. Estos símbolos forman parte del Portal Aragonés de Comunicación Alternativa y están disponibles gratuitamente para todos los especialistas interesados a través de una licencia Creative Commons.

Centrándonos específicamente en los símbolos pictográficos, aunque son bien conocidos en nuestro país y se utilizan en multitud de materiales, cuadernos de comunicación o software para comunicación aumentativa y alternativa (como por ejemplo Araboard, e-Mintza o CPA), sin embargo no se han estudiado sus propiedades formales, especialmente su iconicidad y grado de transparencia. Por tanto, no tenemos información sistemática y controlada que señale si los pictogramas ARASAAC tienen un adecuado grado de transparencia, ni tampoco cómo se comparan con otros conjuntos de símbolos que se utilizan de manera habitual, como el SPC.

Para responder a las cuestiones de cuál es el grado de transparencia de los pictogramas ARASAAC y la relación respecto a otros conjuntos de símbolos, se diseñó un estudio similar al llevado a cabo por Bloomberg et al. (1990) en el que se valora la transparencia mediante la percepción subjetiva de los participantes, utilizando sin embargo una tarea informatizada en la que se comparó la transparencia de distintos símbolos pertenecientes a los conjuntos de ARASAAC, SPC y Bliss, distinguiendo también cuatro tipos de símbolos en función de su categoría gramatical: nombres, verbos, adjetivos, y elementos lingüísticos.

\section{Método}

\section{Sujetos}

Participaron 34 estudiantes universitarios de primer año (29 mujeres, 5 hombres). La edad media era de 18,7 años (desviación típica 1,8 años). Todos eran alumnos de primer y segundo curso del Grado en Logopedia y ninguno tenía conocimiento previo de asignaturas relacionadas con sistemas alternativos y aumentativos de comunicación. 


\section{Estímulos}

Se seleccionaron 38 palabras distintas, correspondientes a cuatro categorías gramaticales: nombres, verbos, adjetivos y elementos linguisticos (es decir, palabras como pronombres o artículos que no tienen carga semántica), como muestra la Tabla 1. La selección de las palabras se hizo siguiendo el trabajo ya mencionado, aunque se añadió la categoría lingüística como elemento novedoso.

Tabla 1. Listado de palabras presentadas en cada conjunto de signos

\begin{tabular}{llll}
\hline Nombres & Verbos & Adjetivos & Lingüísticos \\
\hline Pelota & llegar & grande & porque \\
cama & beber & frio & después \\
libro & dar & sucio & tú \\
caramelo & ir & asustado & antes \\
coche & ayudar & feliz & cuando \\
puerta & abrir & pequeño & cómo \\
niña & jugar & más & dónde \\
tv & leer & no & ellos \\
cuarto de baño & dormir & triste & \\
silla de ruedas & querer & enfermo & \\
\hline
\end{tabular}

Cada una de estas palabras era presentada tres veces, una por cada uno de los conjuntos de símbolos que se utilizaron, de forma que se pudiera comparar la transparencia de los símbolos ARASAAC frente a los SPC, que son considerados los más transparentes, y también frente a los Bliss, que se consideran los más opacos. Para que el lector pueda ver las características de los pictogramas empleados, la Figura 1 muestra los símbolos correspondientes al verbo "ABRIR" en los tres conjuntos. 


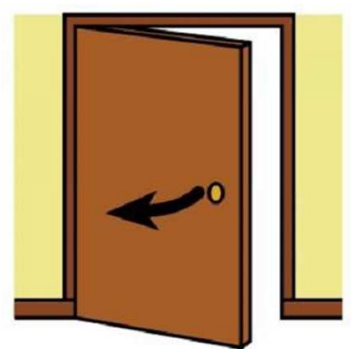

ARASAAC

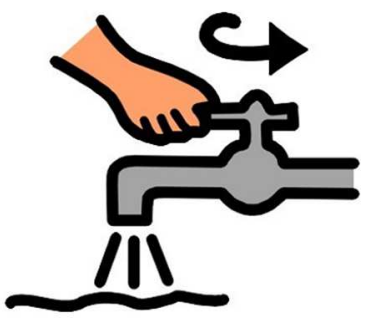

SPC $\wedge$

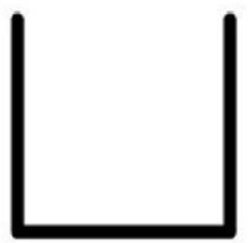

Bliss

Figura 1. Ejemplo de la palabra ABRIR en los tres conjuntos de signos

\section{Aparatos}

Las sesiones se llevaron a cabo de manera individual en una sala equipada con un ordenador PC, un ratón y una pantalla de 14 pulgadas. Se diseñó un programa específico en lenguaje Visual Basic (Cabello, Barnes-Holmes, Stewart y O’Hora, 2002).

\section{Procedimiento}

Todos los participantes fueron expuestos al mismo procedimiento, que comenzaba explicando en qué consistía el experimento mediante un ensayo de prueba. A continuación se iniciaba la tarea, compuesta por un total de 114 ensayos (correspondientes a los 38 símbolos de los tres conjuntos) con la misma estructura: se mostraba uno de los símbolos en la parte central superior de la pantalla con su significado escrito en la base, y un segundo después, aparecía en la parte inferior una escala de valoración tipo Likert de 1 a 7 (ver Figura 2).

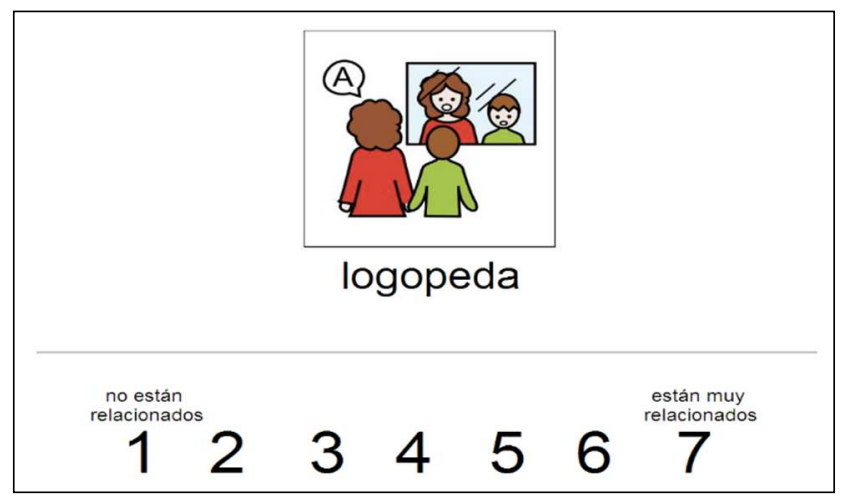

Figura 2. Representación de la estructura de cada ensayo 
El participante debía utilizar el ratón para seleccionar la puntuación de la escala que mejor reflejara la relación entre el símbolo y su significado. Una puntuación de 1 significaba que el pictograma y su significado no estaban relacionados en absoluto, mientras que por el contrario una puntuación de 7 representaba una estrecha relación entre ambos. Los sujetos podían responder sin restricción de tiempo, y tras cada respuesta la pantalla quedaba en blanco y aparecía automáticamente el siguiente símbolo a evaluar, sin ningún tipo de retroalimentación.

Cada uno de los 114 símbolos se presentaba una única vez y en un orden aleatorio para evitar efectos de orden.

\section{Resultados}

En primer lugar se presentan los estadísticos descriptivos de los valores de transparencia. La Tabla 2 muestra las medias y desviaciones típicas para cada uno de los tres conjuntos de símbolos y para cada una de las cuatro categorías gramaticales, entendidos esos valores de transparencia como la valoración subjetiva de la relación entre signos gráficos y referentes.

Tabla 2. Medias y desviaciones típicas de los valores de transparencia

\begin{tabular}{lllllllll} 
& \multicolumn{3}{c}{ Nombres } & \multicolumn{3}{c}{ Verbos } & \multicolumn{3}{c}{ Adjetivos } & \multicolumn{2}{c}{ Lingüísticos } \\
& Med & DT & Med & DT & Med & DT & Med & DT \\
\hline ARASAAC & 6,932 & 0,023 & 6,115 & 0,092 & 6,494 & 0,080 & 4,339 & 0,147 \\
SPC & 6,329 & 0,089 & 5,276 & 0,111 & 6,126 & 0,077 & 4,956 & 0,167 \\
Bliss & 3,262 & 0,165 & 2,165 & 0,137 & 2,053 & 0,133 & 2,431 & 0,141 \\
\hline
\end{tabular}

En el análisis estadístico posterior de los datos, se encontró que el supuesto de esfericidad no se cumplió para los factores principales (conjunto de símbolos y categoría gramatical), por lo que se utilizó como criterio de significación estadística la prueba $\mathrm{F}$ multivariada Lambda de Wilks. El supuesto sí se cumplió para el factor de interacción (conjunto x categoría), y en consecuencia se utilizó como criterio de significación estadística la prueba $\mathrm{F}$ univariada habitual.

Para los efectos del conjunto de símbolos y de la categoría gramatical se obtuvieron diferencias estadísticamente significativas (lambda $=0.027, \quad \mathrm{p}<0.001$; lambda=0.097, p<0.001), lo mismo que para la interacción $(\mathrm{F}=82.907, \mathrm{p}<0.001)$. 
Tras comprobar que había diferencias estadísticamente significativas en el factor de conjunto de símbolos, se realizaron las comparaciones por pares pertinentes, obteniéndose diferencias estadísticamente significativas entre los tres conjuntos de pictogramas (dif SPC-ARASAAC $=-0.298, \mathrm{p}<0.001$; dif SPC-Bliss = 3.194, $\mathrm{p}<0.001$; dif ARASAAC-Bliis = 3.492, p<0.001). Respecto al factor categoría gramatical, también se obtuvieron diferencias estadísticamente significativas entre las cuatro categorías (dif nombres-verbos $=0.989, \mathrm{p}<0.001 ;$ dif nombres-adjetivos $=0.617, \mathrm{p}<0.001 ;$ dif nombreslingüísticos $=1.500, \mathrm{p}<0.001 ;$ dif verbos-adjetivos $=-0.373, \mathrm{p}<0.001 ;$ dif verboslingüísticos $=0.610, \mathrm{p}<0.001 ;$ dif adjetivos-lingüísticos $=0.982, \mathrm{p}<0,001)$. Por último, la interacción también arrojó diferencias estadísticamente significativas en todas las comparaciones por pares dos a dos.

Estos hallazgos pueden representarse gráficamente en la Figura 3 que muestra el perfil de las medias marginales estimadas.

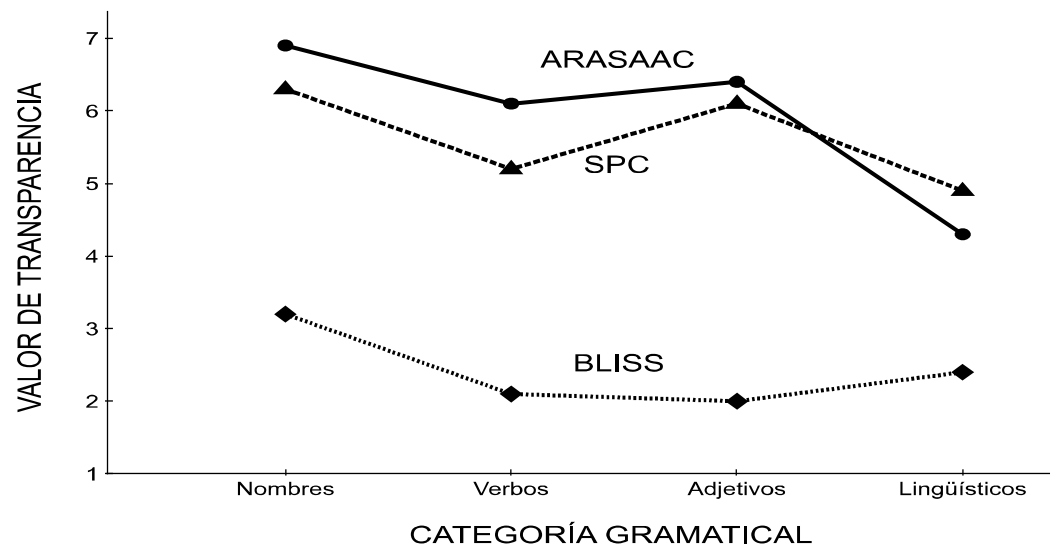

Figura 3. Media de los valores de transparencia para cada conjunto de signos y categoría gramatical

Los símbolos Bliss obtienen las peores valoraciones de transparencia en todas las categorías, mientras que los símbolos SPC son significativamente más transparentes. En cuanto a los símbolos ARASAAC, encontramos que son a su vez significativamente más transparentes que los SPC en nombres, verbos y adjetivos, pero destaca que esta superioridad no aparece en la categoría de símbolos lingüísticos.

Si nos centramos en las diferencias entre categorías gramaticales, se encuentra que la categoría de nombres tiene la mayor transparencia, seguida de adjetivos y verbos, existiendo diferencias significativas entre todas ellas. Por último, la categoría de símbolos 
lingüísticos es la que muestra peor transparencia y donde el significado de los pictogramas resulta menos evidente.

\section{Discusión}

Los objetivos fundamentales de este trabajo fueron (a) examinar el grado de transparencia e iconicidad de los símbolos pictográficos de ARASAAC y (b) comparar esas características con las de otros conjuntos de símbolos bien establecidos en el campo de la comunicación alternativa. En este sentido, los datos señalan que los pictogramas ARASAAC tienen un grado de transparencia adecuado, y que su significado resulta más claro incluso que el de los símbolos SPC, que en la mayoría de estudios previos aparecen como los más transparentes (por ejemplo Mizuko y Reichle, 1989). Por tanto y de acuerdo a la "hipótesis de la iconicidad", los pictogramas ARASAAC tienen un significado que es fácil de entender y aprender, y su uso está justificado en el marco de la comunicación aumentativa y alternativa.

Del análisis de los resultados también se desprende que el grado de transparencia varía en función de la categoría gramatical de los símbolos, lo cual coincide con evidencias previas que señalan que los símbolos correspondientes a nombres son más fáciles de aprender que los relativos a otros tipos de palabras como verbos, adjetivos, etcétera (Mirenda y Locke, 1989; Mizuko, 1987; Schlosser et al, 2012). En este sentido, hay que destacar que en este trabajo se presta atención a palabras lingüísticas como pronombres o artículos, que han recibido menos atención en la literatura (Schlosser y Sigafoos, 2002) y que han arrojado hallazgos interesantes al ser la única categoría gramatical en la que los símbolos SPC son mejores que los símbolos ARASAAC. Esto significa que al estudiar las características de transparencia de los símbolos, no basta con un análisis amplio (SPC mejor que Bliss, SPC peor que ARASAAC) sino que hay que hacer una aproximación mucho más detallada, ya que en caso contrario se pueden escapar resultados de interés y con posibles implicaciones para la labor clínica a la hora de elegir un conjunto de símbolos u otro. Futuros estudios podrían orientarse a continuar investigando esta aparente desventaja de los pictogramas ARASAAC en esta categoría concreta.

Por último, debe destacarse que el procedimiento empleado en este estudio supone una mejora sobre el original de Bloomberg et al. (1990), ya que en en ese trabajo los sujetos disponían de un cuadernillo donde estaban impresos todos los símbolos en un orden fijo y en el que podían enmendar sus respuestas iniciales, lo que podría generar 
efectos de orden que aquí se controlan a través de una tarea informatizada y de la presentación aleatoria de los símbolos.

En cuanto a limitaciones del trabajo, destacan dos. La primera es que constituye un estudio inicial con sujetos adultos y sin patología, de manera que cualquier inferencia a partir de los resultados debe considerarse con precaución. No obstante, en la literatura que compara distintos conjuntos de signos se encuentra una homogeneidad entre distintas poblaciones, de manera que aquellos con mayor o menor transparencia la demuestran tanto en adultos como en niños con y sin patología; por ejemplo, los mismos resultados de Bloomberg et al. (1990) con adultos sin patología se encontraron en adultos y niños con discapacidad intelectual (Mirenda y Locke, 1989; Mizuko y Reichle, 1989). Por tanto, es de esperar que los hallazgos de este trabajo se repitan también en otros grupos de participantes, aunque debido a que la transparencia en símbolos gráficos es un aspecto complejo que puede depender de múltiples variables (véase, por ejemplo, Beukelman y Mirenda, 2013) la replicación de los resultados es una cuestión empírica que deberá comprobarse en futuros estudios.

Y la segunda es que se utiliza una escala tipo Likert para valorar el grado de transparencia de manera subjetiva, lo cual puede resultar más complicado que otros procedimientos en los que se elige directamente y de forma más objetiva entre distintos símbolos similares. En cualquier caso, se están realizando nuevos estudios para replicar los resultados en niños con desarrollo normal y distintas patologías, así como con un procedimiento de selección más directo similar al utilizado por Mizuko (1987) o por Musselwhite y Ruscello (1984).

En el que puede considerarse el trabajo seminal sobre comunicación alternativa en España, Tamarit (1989) ya señalaba la necesidad de realizar investigación dentro de este campo. Aunque de forma inicial, esta investigación constituye la primera aportación empírica al estudio de los símbolos de ARASAAC y arroja evidencias sobre sus características como conjunto pictográfico válido. En este sentido, hay que destacar que los símbolos parecen ser adecuados (incluso más que los de SPC para la mayoría de categorías gramaticales) y que por tanto se recomienda su utilización, pero que esta afirmación se hace por primera vez en base a datos empíricos y no a preferencias o suposiciones subjetiva. Esa es la principal aportación del trabajo y muestra la importancia de la investigación aplicada al terreno de la comunicación alternativa. 


\section{Referencias}

Angermeier, K., Schlossoer, R., Luiselli, J., Harrington, C., y Carter, B. (2008). Effects of iconicity on requesting with the picture exchange communication system in children with autism spectrum disorder. Research in Autism Spectrum Disorders, $2,430-446$.

Beukelman, D., y Mirenda, P. (2013). Augmentative and alternative communication: Supporting children and adults with complex communication needs. Baltimore, MA: Paul H. Brookes Publishing Co.

Bloomberg, K., Karlan, G., y Lloyd, L. (1990). The comparative translucency of initial lexical items represented in five graphical symbol systems and sets. Journal of Speech and Hearing Research, 33, 717-725.

Cabello, F., Barnes-Holmes, D., Stewart, I. y O’Hora, D. (2002). Visual Basic in the Experimental Analysis of Behavior. Experimental Analysis of Human Behavior Bulletin, 20, 17-20.

Fuller, D. (1997). Effects of translucency and complexity on the associative learning of Blissymbols by cognitively normal children and adults. Augmentative and Alternative Communication, 12, 30-39.

Fuller, D., y Lloyd, L. (1997). Symbol selection. En L. Lloyd, D. Fuller y H. Arvidson (Eds.), Augmentative and alternative communication: Handbook of principles and practice (pp. 214-225). Needham Heights, MA: Allyn \& Bacon.

Fuller, D., Lloyd, L., y Stratton, M. (1997). Aided AAC symbols. En L. Lloyd, D. Fuller y H. Arvidson (Eds.), Augmentative and alternative communication: Handbook of principles and practice (pp. 48-79). Needham Heights, MA: Allyn \& Bacon.

Hetzroni, O. E., Quist, R., y Lloyd, L. (2002). Translucency and complexity: Effects on Blissymbol learning using computer and teacher presentations. Language, Speech, and Hearing Service in Schools, 33, 291-303.

Hulburt, B., Iwata, B. y Green, J. (1982). Nonvocal language acquisition in adolescents with severe physical disabilities: Blissymbols versus iconic stimulus formats. Journal of Applied Behavior Analysis, 15, 241-258.

Koul, R., Schlosser, R., y Sancibrian, S. (2001). Effects of symbol, referent, and instructional variables on the acquisition of aided and unaided symbols by individuals with Autism Spectrum Disorders. Focus on Autism and Other Developmental Disabilities, 16, 162-176. 
Luftig, R., y Bersani, H. (1985). An investigation of two variables influencing Blissymbol learnability with nonhandicapped adults. Augmentative and Alternative Communication, 1, 32-33.

Mirenda, P., y Locke, P. (1989). A comparison of symbol transparency in nonspeaking children with intellectual disabilities. Journal of Speech and Hearing Disorders, $54,131-140$.

Mizuko, M. (1987). Transparency and ease of learning of symbols represented by Blissymbolics, PCS, and Picsyms. Augmentative and Alternative Communication, $3,129-136$.

Mizuko, M., y Reichle, J. (1989). Transparency and recall of symbols among intellectually handicapped adults. Journal of Speech and Hearing Disorders, 54, $627-633$.

Musselwhite, C., y Ruscello, D. (1984). Transparency of three communication symbol systems. Journal of Speech and Hearing Research, 27, 436-443.

Schlosser, R., y Sigafoos, J. (2002). Selecting graphic symbols for an initial request lexicon: Integrative review. Augmentative and Alternative Communication, 18, $102-123$.

Schlosser, R., Shane, H., Sorce, J., Koul, R., Bloomfield, E., Debrowski, L., DeLuca, T., Miller, S., Schneider, D. y Neff, A. (2012). Animation of graphic symbols representing verbs and prepositions: Effects on transparency, name agreement, and identification. Journal of Speech, Language, and Hearing Research, 55, 342-358.

Tamarit, J. (1989). Uso y abuso de los sistemas de comunicación. Comunicación, Lenguaje y Educación, 1, 81-94.

Wilkinson, K., y Jagaroo, V. (2004). Contributions of principles of visual cognitive science to AAC system display design. Augmentative and Alternative Communication, 20, 123-136. 